Revue de droit comparé du travail et de la sécurité sociale

3 | 2020

La Directive 2019/1158 du 20 juin 2019 concernant l'équilibre entre vie personnelle et vie privée des parents et des aidants

\title{
Le droit à une pension de survie en République de Serbie
}

Filip Bojić

\section{(2) OpenEdition \\ Journals}

Édition électronique

URL : https://journals.openedition.org/rdctss/1041

DOI : $10.4000 /$ rdctss. 1041

ISSN : 2262-9815

Éditeur

Centre de droit comparé du travail et de la sécurité sociale

Édition imprimée

Date de publication : 1 novembre 2020

Pagination : 222-225

ISSN : 2117-4350

Référence électronique

Filip Bojić, "Le droit à une pension de survie en République de Serbie », Revue de droit comparé du travail et de la sécurité sociale [En ligne], 3 | 2020, mis en ligne le 01 novembre 2021, consulté le 11 novembre 2021. URL : http://journals.openedition.org/rdctss/1041; DOI : https://doi.org/10.4000/ rdctss. 1041

\section{(c) (i) $\odot$}

Revue de droit comparé du travail et de la sécurité sociale est mise à disposition selon les termes de la Licence Creative Commons Attribution - Pas d'Utilisation Commerciale - Pas de Modification 4.0 International. 


\title{
FILIP BOJIĆ
}

\author{
UNIVERSITÉ DE BELGRADE
}

\section{LE DROIT À UNE PENSION DE SURVIE EN RÉPUBLIQUE DE SERBIE}

Le droit à une pension de survie est l'un des droits fondamentaux garantis aux membres de la famille de l'assuré décédé, dont les conditions sont déterminées par la Loi sur l'assurance retraite et invalidité de la République de Serbie adoptée en 2003.

Cette année, l'Assemblée nationale a adopté un grand nombre d'amendements liés à l'exercice du droit à une pension de survie, dans le prolongement d'amendements déjà votés en décembre $2019^{1}$ qui prévoient que des conjoints non mariés peuvent désormais exercer le droit à une pension de survie, sous certaines conditions, ce qui n'était pas le cas auparavant dans la législation serbe. Jusqu'alors, les conjoints non mariés ne bénéficiaient en effet d'aucun droit à une pension de survie, quelle quait été la durée de leur vie commune.

Outre la mise en conformité de la loi avec la Constitution serbe, la ratio legis de ces amendements résidait dans la recherche de l'équité entre les unions, qu'elles soient conjugales ou concubines, d'une part et d'autre part dans l'augmentation du nombre de personnes âgées pouvant avoir droit à une pension de survie, afin notamment d'améliorer leur situation économique dans la société2.

Il faut souligner que la pension de survie dispose d'une longue tradition dans la législation serbe. Les premières formes sont apparues au milieu du XIXe siècle et, au départ, consistaient en pensions garanties aux veuves et aux enfants de fonctionnaires décédés, puis aux veuves et aux enfants d'enseignants. Avant l'adoption des amendements de 2019-2020, la loi prévoyait que les titulaires du droit à une pension de survie, outre les membres de la famille proche de l'assuré décédé - c'est-à-dire du bénéficiaire de la pension de retraite, tels qu'un conjoint et des enfants, qu'ils soient nés ou non au sein du mariage, etc.), pouvaient également être les membres de la famille élargie du défunt - comme ses parents, frères ou sœurs, etc. ${ }^{3}$. II convient toutefois de noter que les membres de la famille élargie n'ont droit à une pension de survie qu'à la condition qu'il n'existe aucun membre de la famille proche.

1 la Loi sur l'assurance retraite et invalidité de la République de Serbie (Journal officiel de la République de Serbie, $n^{\circ} 34 / 2003$; Modifications législatives n64/2004, n84/2004, $n^{\circ} 85 / 2005, n^{\circ 101 / 2005,} n^{\circ} 63 / 2006, n^{\circ} 5 / 2009, n^{\circ} 107 / 2009, n^{\circ} 101 / 2010, n^{\circ} 93 / 2012$, $n^{\circ} 62 / 2013, n^{\circ} 108 / 2013, n^{\circ} 75 / 2014, n^{\circ} 142 / 2014, n^{\circ} 73 / 2018, n^{\circ} 46 / 2019$.

2 Voir la proposition de loi sur des amendements de La Loi sur l'assurance retraite et invalidité, explication p.41 : http://www.parlament.gov.rs/upload/archive/files/cir/pdf/predlozi zakona/ 2019/2666-19.pdf

3 Loi sur l'assurance retraite et invalidité de 2003, art. 34. 


\section{République de Serbie}

Les amendements de la Loi sur l'assurance retraite et invalidité de décembre 2019 ont permis de considérer les concubins comme des membres à part entière de la famille proche de l'assuré.

Il est important de souligner que, dans la législation serbe, un membre de la famille de l'assuré décédé peut avoir le droit à une pension de survie uniquement si les conditions requises, précisées dans la loi, étaient remplies par le défunt. Ainsi, le droit à une pension de survie sera exercé par les membres de la famille dont le défunt a cotisé au minium pendant 5 ans, ou a rempli les conditions relatives à l'octroi d'une pension de vieillesse - pension prématurée de vieillesse ou pension $d^{\prime}$ 'invalidité. Cependant, si le décès fait suite à un accident du travail ou à une maladie professionnelle, les membres de sa famille acquièrent le droit à une pension de survie quelle que soit la durée de cotisation de l'assuré défunt ${ }^{4}$.

Il subsistait toutefois une controverse concernant l'exercice du droit à une pension de survie en République de Serbie puisque, pendant des années, ce droit n'a pas été reconnu aux conjoints non mariés, bien que la Constitution serbe de 2006 et la loi sur le droit de la famille de 2005 aient reconnu aux conjoints les mêmes droits qu'aux partenaires mariés ${ }^{5}$. Par conséquent, en 2008, la Cour constitutionnelle s'est saisie de la question de l'inconstitutionnalité de certaines dispositions de la Loi sur l'assurance retraite et invalidité, déclarant notamment l'incompatibilité de certaines de ses dispositions avec la Constitution en ce qu'elles discriminaient les personnes vivant en concubinage par rapport aux personnes mariées. Toujours d'après la Cour, ces dispositions sont également en conflit avec les normes constitutionnelles qui garantissent l'égalité de l'union conjugale et non conjugale devant la loib. Cependant, la Cour a conclu qu'il n'y avait aucune raison d'examiner cette question dans la mesure où elle ne disposait pas de la compétence nécessaire pour prendre une quelconque décision sur le sujet. Selon la Cour, il n'existait pas de motif suffisant pour engager une procédure car elle n'avait ni le rôle, ni les pouvoirs d'un législateur, mais disposait uniquement du pouvoir d'abroger la norme existante ${ }^{7}$.

Néanmoins, la Cour s'est engagée à adresser une lettre à l'Assemblée nationale, dans laquelle elle soulignait la nécessité de réviser les dispositions litigieuses de la loi sur l'assurance pension et invalidité relatives au droit à une pension de survie pour le conjoint survivant, qu'il soit marié et non marié, conformément à la Constitution et aux actes internationaux ${ }^{8}$.

4 Loi sur l'assurance retraite et invalidité de 2003, art. 27.

5 Constitution de la République de Serbie, Journal officiel de la République de Serbie, $n^{\circ} 98 / 2006$, art. 62, acte 5; Loi sur le droit de la famille, Journal officiel de la République de Serbie, $n^{\circ} 18 / 2005, n^{\circ} 72 / 2011$ et $n^{\circ} 6 / 2015$, art. 4, acte 2.

6 Voir la décision de la Cour constitutionnelle de Serbie n IУ 3 90/2008 du 30 juin 2011 qui rejette l'initiative de la révision constitutionnelle des art. 28, 29, 30 et 34 de La Loi sur l'assurance retraite et invalidité.

7 Décision de la Cour constitutionnelle de RS de rejet de l'initiative de révision constitutionnelle, art. 28, 29, 30 et 34 de La Loi sur l'assurance retraite et invalidité, $n^{\circ} \mid Y_{3}$ 90/2008 du 30 juin 2011.

8 Décision de la Cour constitutionnelle de RS de rejeter l'initiative de la révision constitutionnelle, art. 28, 29, 30 et 34 de La Loi sur l'assurance retraite et invalidité, n IУ 3 90/2008 du 30 juin 2011. 
C'est donc une décennie plus tard, en décembre 2019, que l'Assemblée nationale a pris en compte la recommandation de la Cour constitutionnelle, en adoptant de nouvelles dispositions reconnaissant le droit à une pension de survie aux conjoints non mariés. Conformément aux règles régissant les relations familiales, ces nouvelles dispositions prévoient également que les conjoints non mariés sont considérés comme des membres à part entière de la famille de l'assuré décédé?.

Ces modifications législatives régissant l'assurance retraite et invalidité en République de Serbie, comme branche distincte de la sécurité sociale, ont ainsi permis aux conjoints non mariés de devenir pleinement égaux aux conjoints mariés, ce qui est somme toute logique et dans la parfaite continuité des normes constitutionnelles en vigueur.

Néanmoins, des conditions supplémentaires liées à la durée de l'union entre partenaires non mariés ont été fixées. II est ainsi prévu que le concubin puisse exercer le droit aux prestations de survivant à la condition que son union avec l'assuré décédé ait duré au moins trois ans, ou qu'un enfant soit né de cette union. Cette même condition d'ancienneté de l'union est requise pour les conjoints mariés dans la mesure où le pays constate de plus en plus de mariages de complaisance, conclus entre un homme âgé et une très jeune femme, permettant à cette dernière de bénéficier d'une pension de survie après la mort de l'assuré.

En outre, les amendements de la Loi sur l'assurance retraite et invalidité prévoient que le conjoint divorcé ou séparé peut bénéficier du droit à une pension de survie dès lors que la Cour lui a préalablement attribué le droit à une pension alimentaire $^{10}$. Ces modifications législatives ont donc parfaitement harmonisé les conditions liées à l'exercice du droit à une pension de survie pour les veufs et veuves de conjoints, qu'ils soient ou non mariés. De plus, il est envisagé que la veuve puisse systématiquement avoir droit à une pension de survie dès lors qu'elle a atteint l'âge de 53 ans, indépendamment de la durée de cotisation de l'assuré défunt, permettant ainsi d'exercer ce droit même si le conjoint survivant a été au chômage toute sa vie ${ }^{11}$. D'autre part, la veuve a droit à une pension de survie si, au moment du décès de son conjoint ou concubin, ou dans un délai d'un an après le décès, elle se trouve dans l'incapacité totale de travailler. Ce même droit lui est reconnu lorsqu'elle a eu un ou plusieurs enfants avec le défunt et qu'elle accomplit personnellement les devoirs parentaux envers ces enfants. Par ailleurs, la loi dispose que la veuve n'ayant pas encore atteint l'âge de 53 ans au moment du décès de son conjoint ou concubin, mais ayant toutefois au moins 45 ans, pourra exercer le droit à une pension dès l'âge de 53 ans.

Le veuf acquiert droit à une pension de survie dans des conditions similaires à celles de la veuve. La seule différence est qu'un veuf a droit à une pension de survie s'il atteint 58 ans avant la mort de sa conjointe ou de sa conjointe non mariée, soit 5 ans de plus que pour les veuves ${ }^{12}$. De plus, la loi ne prévoit pas la possibilité qu'un veuf puisse exercer son droit ultérieurement, comme prévu pour les veuves, c'est-à-dire

9 Loi sur l'assurance retraite et invalidité de 2003, art. 28, acte 1, § 1.

10 Loi sur l'assurance retraite et invalidité de 2003, art. 28.

11 lbid., art. 29.

12 Loi sur l'assurance retraite et invalidité de 2003, art. 30. 


\section{Républioue de SERBie}

dès lorsqu'il atteint l'âge de 58 ans et dans l'hypothèse où il aurait au moins 45 ans au moment du décès de sa conjointe. En effet, étant donné que, ces dernières années, la République de Serbie connaît un taux de chômage élevé chez les hommes de plus de 50 ans, il est probable que le législateur soit enclin à envisager des règles plus strictes que pour les veuves ${ }^{13}$.

13 Pendant des années, les veufs se trouvaient en situation d'inégalité par rapport aux veuves concernant l'exercice de droit à une pension de survie. Dans un premier temps, seule la veuve avait droit à une pension de survie et ce droit n'a pas été reconnu au veuf pendant des années. Voir Convention de l'Organisation internationale du travail concernant la sécurité sociale (norme minimum), en cas de décès du soutien de famille, le droit aux prestations sociales est prévu pour les veuves et les enfants, ce qui confirme une fois de plus qu'une révision de cette norme internationale est nécessaire. 